\title{
Comunidades de desarrolladores, educación e innovación
}

\section{Sumario}

1. Introducción

2. Las comunidades y sus estructuras

3. Comunidades, educación e innovación

4. Google y las comunidades de desarrolladores

5. Conclusiones

Fecha de entrada: 11-12-2014

Fecha de aceptación: 23-12-2014

\section{Extracto:}

Andrés L. Martínez Ortiz, también llamado Almo, es gerente del Programa para Desarrolladores en la Organización de Ingeniería de Google. Él se centra en impulsar el éxito de productos para desarrolladores de Google y la web abierta mediante la creación de un próspero ecosistema de aplicaciones y de las empresas integradas. En estrecha colaboración con Gestión de Producto, Marketing, Relaciones Públicas y Desarrollo Empresarial, trabaja y apoya a las comunidades de desarrolladores, iniciando actividades que satisfagan las necesidades del ecosistema de innovación. Además, se reúne con desarrolladores y socios en grandes compañías y compañías recientemente creadas, universidades y empresas, promocionando los estándares abiertos y tecnologías de Google.

Palabras claves: desarrollo, Google, tecnología del software, comunidades de desarrolladores, innovación.

\footnotetext{
A. L. Martínez Ortiz, gerente del Programa para Desarrolladores en la Organización de Ingeniería de Google.
} 


\section{Developer communities, education and innovation}

\section{Abstract:}

Andrés L. Martínez Ortiz, a.k.a Almo, is Developer Program Manager in Google's Engineering Organisation. He is focused on driving the success of Google's developer products and the open web by creating a thriving ecosystem of 3rd party applications and businesses built on them. Closely with Product Management, Marketing, Public Relations, Business Development, and other, he works with and supports developer communities, initiating activities that meet the needs of the innovation ecosystem. In addition, he meets with developers and partners in large companies, startups, universities and enterprises, promoting open standards and Google technologies.

Keywords: development, Google, software technology, developer communities, innovation.

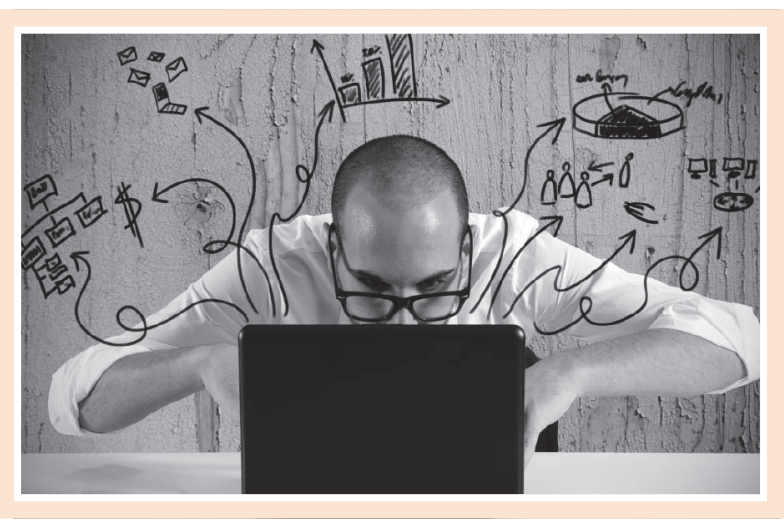

\section{INTRODUCCIÓN}

Durante los últimos treinta años, las redes de computadoras e internet, así como los esquemas de licenciamiento que favorecen la colaboración, han revolucionado la forma en la que se aprende, se desarrolla y se innova en la tecnología software. Recientemente, otras áreas tecnológicas como makers o biocoders han adoptado con éxito el modelo.

Pero es más, disciplinas como las humanidades digitales ponen de manifiesto, en estrecha correspondencia con las comunidades actuales, el sustrato social que articula el desarrollo de la cultura, el arte o la economía. Algunos autores van más allá y afirman, incluso, que la filosofía occidental surgió de redes sociales establecidas ya desde épocas presocráticas que articularon el desarrollo del pensamiento moderno.

La reciente publicación del libro Social physics constituye un importante paso que va desde las líneas de investigación de ciencias sociales a la consolidación de una disciplina que fundamenta gran parte de las experiencias que se encuentran en comunidades de desarrolladores de software.

En tecnología, es ahora cuando empezamos a elaborar modelos explicativos que permiten entender por qué en el desarrollo de las ideas no solo se depende de aquellas mentes más brillantes, sino también de su capacidad para crear comunidades a su alrededor, convirtiendo las ideas en estrategias y la innovación en el flujo social de las mismas.

Dentro del Programa de Desarrolladores de Google, estas tendencias son reconocidas y aprovechadas, utilizándose una estrategia comunitaria en programas de apoyo a desarrolladores de software como son los Grupos de Desarrolladores de Google (GDG) o los Google Developer Experts (GDE). 


\section{LAS COMUNIDADES Y SUS ESTRUCTURAS}

Las comunidades de desarrolladores presentan una enorme cantidad de variantes, que van desde el desarrollador individual, que publica su código en agregadores como GitHub o BitBucket, a grandes comunidades multiproyecto, como Debian o Apache, que abordan el desarrollo de piezas de software de enorme complejidad.

Desde un punto de vista estructural, las comunidades se sustentan en dos aspectos fundamentales: las licencias del software y las plataformas on-line de colaboración. Los esquemas de licenciamiento abierto establecen los términos que permiten la ejecución del software, su copia, modificación y distribución, mientras que las plataformas on-line soportan los procesos y la interacción social de la comunidad.

Los procesos y su evolución dinámica permiten categorizar las comunidades, ofreciendo una visión del esquema de incentivos o producto obtenido por los miembros. Permiten responder a la siguiente pregunta: ¿por qué los desarrolladores participan en la comunidad? En términos generales existen tres categorías de procesos: compartir/curar, colaborar y cooperar. La gran mayoría de las comunidades evolucionan a un ente que integra en mayor o menor medida todas ellas, siguiendo una estructura jerarquizada.

En las comunidades en las que el proceso principal es compartir/curar, el grueso de sus miembros tiene un perfil bajo de participación, y una parte reducida de los mismos se centran en dos procesos: comparten contenidos y/o los evalúan o curan, produciendo como resultado información filtrada. Inteligencia de grupo. Este sería el principal incentivo de la comunidad en el modelo presente en la mayoría.

El tránsito de un esquema de comunidad a otra se produce, normalmente, a través de un proceso de maduración, entendido como la evolución desde comunidades con bajo perfil de participación a otras en las que sus miembros tienen un mayor nivel de implicación, llevando a cabo procesos de colaboración, para en una fase posterior, al incorporar visión estratégica, convertir estos en procesos de cooperación. Este sería el estado de máxima evolución.

\section{Los alumnos que participan activamente en comunidades de desarrolladores mejoran su empleabilidad y adquieren experiencia profesional temprana}

\section{COMUNIDADES, EDUCA- CIÓN E INNOVACIÓN}

Esta dinámica permite que las comunidades impulsen un particular modelo de innovación, que va desde el aprendizaje de las tecnologías software, hasta su desarrollo e innovación estratégica. En numerosos casos, también se produce un último paso que lleva ciertas innovaciones al mercado, al desarrollar los correspondientes modelos de negocio, en estrecha colaboración con el ecosistema de emprendedores.

En lo que respecta a la formación, las comunidades juegan un papel doble. Por un lado, complementan la formación reglada, acercando la industria a las aulas, y constituyen un entorno de hands-on en el que el foco reside en hacer y evaluar. Al carecer de barreras de acceso, flexibilizan la educación superior, resultando por tanto un excelente complemento a la educación reglada. Como resultado de todo ello, los alumnos que participan activamente en comunidades de desarrolladores mejoran su empleabilidad y adquieren experiencia profesional temprana. Por otro lado, constituyen la vía preferida por multitud de profesionales para acceder a la formación continua. Esta es especialmente importante en el campo de la tecnología software, centro de numerosos cambios durante los últimos años. Numerosas comunidades de desarrolladores están integradas por profesionales que llevan a cabo grupos de estudio, análisis de rendimiento, documentan patrones de diseño o depuran errores de plataformas novedosas, que cuentan con amplia implantación en el mercado y carecen de fuentes alternativas de formación. Como resultado, se mejoran los procesos de transferencia tecnológica, reduciendo los tiempos de adopción e incrementando su impacto en la industria. 


\section{GOOGLE Y LAS COMUNIDADES DE DESARROLLADORES}

Todas estas razones hacen de las comunidades de desarrolladores un mecanismo excepcional para implementar acciones de difusión de tecnología software. En particular, dentro de Google se utilizan para compartir y curar contenidos y acciones de formación y para incrementar la densidad crítica de desarrolladores excelentes de alto impacto en la comunidad.

Los GDG son comunidades que tienen como objetivo compartir y curar contenidos formativos y acciones que mejoren el conocimiento en tecnologías de Google y plataformas abiertas. Desde el punto de vista tecnológico, tienen un enfoque horizontal, acogiendo a desarrolladores diversos y con distinto nivel de maduración.

Con base geográfica, los diferentes grupos se integran de forma orgánica en estructuras nacionales e internacionales, con las que se persiguen metas más ambiciosas que requieren colaboración y cooperación. Gracias a esta estructura, miles de desarrolladores realizan eventos de difusión y promoción de la tecnología a lo largo de todo el mundo.

Con el programa GDG, en cambio, desarrolladores con un alto nivel de excelencia técnica que realizan labores de difusión tecnológica se integran en una comunidad con el fin principal de favorecer la densidad crítica. Con un foco absolutamente vertical, los GDE están asociados a la tecnología de la que son expertos, teniendo el foco geográfico una menor importancia.

Como resultado, estos desarrolladores se convierten en un referente para la comunidad global, se asumen retos de desarrollo de software más ambiciosos y se establece una relación de mecenazgo con desarrolladores menos experimentados.

Alentados por los satisfactorios resultados obtenidos con estos programas, aproximaciones similares están siendo evaluadas en sectores tecnológicos más especializados u orientados a producto y también en el campo de los ecosistemas de emprendedores. Esperamos poder compartir pronto estas experiencias.

\section{(...) las comunidades de desarrolladores constituyen uno de los principales agentes del desarrollo y la innovación de la tecnología software}

\section{CONCLUSIONES}

En definitiva, las comunidades de desarrolladores constituyen uno de los principales agentes del desarrollo y la innovación de la tecnología software. Han sido el referente utilizado por muchas otras disciplinas para sacar ventaja de las comunicaciones e internet y han ayudado a acercar industria y academia, comunidades que para implementar con éxito procesos de innovación deben trabajar estrechamente.

La participación en comunidades de desarrolladores tiene un beneficioso efecto en estudiantes y profesionales del desarrollo de software. Google implementa con comunidades estrategias de mejora de la formación, así como de incremento de la densidad crítica, cohesionando las comunidades. 